\title{
Toxoplasmosis cerebral asociado a VIH-SIDA: Revisión de literatura y reporte de un caso
}

\author{
Zairie, Niguelie Cawich Santiago Hernández Bojorge, \\ Olíz Solís Carbajal, Clara González Moncada. \\ Autores correspondientes: Tel. (505) 8406925/83768779. \\ Correo electrónico: equinvest3@yahoo.com
}

\section{RESUMEN}

La toxoplasmosis cerebral es la infección oportunista más común del SNC en pacientes con SIDA. Es la causa del 10 al $50 \%$ de las lesiones ocupantes de espacio relacionadas con el HIV. La presentación de una lesión toxoplásmica cerebral como primera manifestación del SIDA no ha sido publicada aún en nuestro país. La toxoplasmosis es una enfermedad oportunista causada por el parásito Toxoplasma gondii, que puede ser vehiculizado a través del consumo de carne cruda o mal cocida, con presencia de quistes o al ingerir alimentos y agua contaminados con ooquistes. Debido a la asociación de esta parasitosis con el síndrome de inmunodeficiencia humana, actualmente es una enfermedad de gran impacto en la población, debido a que ocasiona una presentación clínica más seria de la enfermedad, con compromiso orgánico múltiple y altas tasas de mortalidad. Se describe el caso de un paciente masculino de 28 años de edad con toxoplasmosis cerebral asociada a virus de la inmunodeficiencia humana (VIH) estado C3 y convulsiones con hemiplejia del hemicuerpo izquierdo.

Palabras clave: Toxoplasmosis, Toxoplasma gondii, VIH,SIDA.

\section{SUMMARY}

Cerebral toxoplasmosis is the most common opportunistic CNS infection in AIDS patients. It is the cause of 10 to $50 \%$ of space-occupying lesions related to HIV. The presentation of a brain injury toxoplasmosis as the first manifestation of AIDS has not yet been published in our country. Toxoplasmosis is an opportunistic disease caused by the parasite Toxoplasma gondii, which can be vehiculizado through the consumption of raw or undercooked meat, with the presence of cysts or by ingesting contaminated food and water with oocysts. Because of the association of this parasitic infection with human immunodeficiency syndrome, it is now a disease of great impact on the population, because it causes a more serious clinical presentation of the disease, with multiple and high mortality rates organ involvement. the case of a male patient 28 years old with cerebral toxoplasmosis associated with human immunodeficiency virus (HIV) status C3 and convulsions with hemiplegia left hemicuerpo described.

Keywords: Toxoplasmosis, Toxoplasma gondii, HIV, AIDS 


\section{INTRODUCCIÓN}

La toxoplasmosis es una infección producida por Toxoplasma gondii (T. gondii), protozoo intracelular de la subclase Coccidia con amplia distribución en todo el mundo. Se estima que alrededor de un tercio de la población mundial tiene anticuerpos contra este parasito, con variaciones regionales de seroprevalencia. En las personas inmunocompetentes, la infección primaria es generalmente asintomática, pero persiste en estado latente durante toda la vida (David Botero, 2012). Cuando existe inmunosupresión se puede desarrollar dos tipos de enfermedad: la infección primaria severa y la infección crónica que se recrudece.

El virus de la inmunodeficiencia humana (HIV) provoca una disminución continua de linfocitos $\mathrm{CD} 4+$, causando un deterioro lentamente progresivo del sistema inmunológico en las personas infectadas. Una de las consecuencias de este hecho es la aparición de infecciones oportunistas con una importante morbilidad y mortalidad. La toxoplasmosis constituye generalmente una complicación tardía en los pacientes infectados con este virus y usualmente ocurre en pacientes con menos de 100 células T CD4 por $\mu 1$ (Ivonne Martin-Hernandez, 2003). La infección primaria con $T$. gondii en hospederos inmunodeprimidos resulta en una infección letal diseminada, involucrando diversos órganos y tejidos como tracto gastrointestinal, pulmones, miocardio, cerebro y ojos. Esta infección oportunista aparece aproximadamente en el 15\% de estos pacientes y es más común en individuos que viven en el Caribe y en Francia (Wong S. Y., 1993). En pacientes con SIDA se puede producir una reactivación de la infección latente con $T$. gondii, cuya frecuencia depende de su prevalencia en la población general. Entre el 10-50\% de los pacientes infectados con VIH y con serología positiva para Toxoplasma desarrollan encefalitis toxoplásmica (ET), con casi un 50\% de secuelas neurológicas y cuya mortalidad se acerca al $20 \%$. Estos porcentajes la convierten en la primera infección oportunista más común que afecta al sistema nervioso central (SNC) en pacientes VIH positivos (Gongora-Biachi R. A., 1998).

La mayoría de los casos de toxoplasmosis en enfermos con SIDA que se han reportado se relacionan con infección del sistema nervioso central, afectando el estado mental, la función neurológica, la visión y llegando hasta la muerte (Luft B. J. Hafner R., 1993). 
La toxoplasmosis es una infección rara en los niños infectados por VIH y la forma clínica más frecuente suele ser la toxoplasmosis congénita. El riesgo de toxoplasmosis congénita en niños nacidos de madres doblemente infectadas por $T$. gondii y VIH no está bien establecido; sin embargo, no parece existir una diferencia significativa entre los hijos de la mujer infectada por VIH y las no infectadas. La reactivación de la infección por T. gondii en la madre durante el embarazo representa un factor de riesgo importante para la transmisión intra uterina (Couvreur J., 1994).

\section{Patogenia}

T. gondii tiene un ciclo de vida en el hospedero definitivo conocido como enteroepitelial. En el hombre y otros animales hospederos intermediarios, el parasito hace un ciclo incompleto de reproducción asexuada donde realiza invasiones extraintestinales, frecuentemente a los músculos y al SNC (Llop, 2001).

El daño producido por T.gondii depende del número de taquizoitos que proliferan en las células, de la virulencia de las cepas, el estado puede ocasionar la ruptura de los quistes. La resistencia frente a la infección por $T$. gondii aumenta con la edad, tal es así que los niños desarrollan enfermedad, mientras que los adultos con sistema inmunológico competente presentan anticuerpos contra el parasito, pero permanecen asintomáticos. El parasito tiene antígenos específicos en cada forma de vida que se desarrolla en el huésped. El taquizoito o forma activa tiene antígenos de membrana, citoplasmáticos y de excreción y secreción; estos últimos muy importante para la penetración del microorganismo en las células del huésped (Luna Talavera, 2009).

El parasito penetra la pared intestinal y sigue la vía linfática o hemática, y se disemina a una gran variedad de tejidos. Penetran en las células de forma activa gracias a sus movimientos y a la producción de lisozimas y hialuronidasa; en algunas ocasiones lo hacen por un procedimiento similar a la fagocitosis (Perez Berrios, 2004). Los taquizoitos se reproducen intracelularmente por endodiogénesis, y pasan de célula a célula causándole muerte; esta proliferación constituye la forma activa de toxoplasmosis. En el hospedero inmunodeprimido la respuesta inmune humoral y celular necesaria para controlar la expansión de los taquizoitos está afectada. Este estado inmune alterado conlleva una progresiva destrucción focal que resulta en el fallo del órgano (ejemplo: encefalitis necrotizante, neumonías y miocarditis) (Martin-Hernandez, 2003). 
En el hospedero inmunocompetente incluso después de la respuesta inmunológica efectiva, no se erradican los microorganismos; se forman algunos quistes en estos órganos desde la primera semana de la infección y permanecen latentes toda la vida del huésped. En los pacientes VIH positivos cuando se produce depresión de su sistema inmune, la proliferación activa de los microorganismos puede causar la reactivación de la enfermedad local y su diseminación. La localización de los quistes se encuentra con preferencia en las células del sistema nervioso central, coriorretina y músculos (esquelético y miocardio). Ocasionalmente los quistes pueden romperse y dejar en libertad los bradizoitos; si son muchos los que se rompen se produce una reactivación de la enfermedad, que puede ser localizada o generalizada (Martin-Hernandez, 2003).

\section{Manifestaciones clínicas}

Los síntomas en los pacientes inmunodeprimidos con toxoplasmosis cerebral pueden ser focales o generalizados, siendo la cefalea, la confusión, la fiebre y convulsiones los más importantes. Clínicamente predomina un síndrome compatible con lesiones ocupantes; son comunes además hemiparesias, deficiencias visuales, hipertensión endocraneana, elementos neurológicos focales, compromiso de pares craneanos, trastornos de la conciencia, alteraciones psíquicas y somnolencia. Raramente se encuentra rigidez de la nuca. La sintomatología es variable dependiendo de la localización de la lesión y su número (McCabe R., 1993). La retinitis toxoplásmica en los pacientes con SIDA se presenta como una retinitis necrotizante, con uno o varios focos blanquecinos o blanco-amarillentos de límites borrosos. La retina se haya engrosada, opaca y sus bordes no son granulares, como los típicos de la retinitis por citomegalovirus, sino homogéneos (Frenkel, 1997).

\section{Diagnóstico}

Ante la sospecha clínica de neorotoxoplasmosis se debe solicitar una Tomografía Axial Computarizada (TAC) de cráneo. El hallazgo inmunológico de una o varias lesiones redondeadas, hipodensas con refuerzo anular post-contraste y edema perilesional reafirma la presunción diagnostica (Ciricillo S., 1990). Aunque otras enfermedades pueden dar una imagen similar, son menos frecuentes. Los abscesos toxoplásmicas suelen localizarse en la unión cortico-subcortical y 
ganglio basal, pero pueden verse en otras topografías. Para la confirmación diagnóstica se debe identificar el germen mediante la realización de una biopsia aunque es riesgosa. En la práctica se admite que frente a la sospecha clínico-imagenológica, se inicia el tratamiento específico. La mejoría clínico-radiológica del paciente, en un plazo medio de diez días es un elemento a favor de ese diagnóstico etiológico (Raffi F., 1997). De no tenerse mejoría, se sospecha que la causa de la lesión es otra y está indicado realizar una biopsia por punción aspirativa o a cielo abierto, enviando muestras para estudio al anatomopatólogo y al microbiólogo. La Resonancia Magnética Imagenológica (RMI) es más sensible para detectar pequeñas lesiones que no se visualizan en la TAC (Ammasari A, 1996). Raramente el diagnostico se hace por detección del parasito en sangre, líquidos orgánicos o tejidos. Sin embargo, es posible detectarlo por técnicas histológicas y aislarlo en cultivos celulares o por inoculación al ratón. Por la técnica de Acción en Cadena de la Polimerasa (PCR) puede detectarse el ADN de T. gondii en tejidos y fluidos corporales. Otras técnicas diagnósticas son Inmunofluorescencia indirecta, ELISA, aglutinación directa, hemaglutinación indirecta, prueba con látex, la prueba de la Toxoplasmina y la prueba de SabinFeldman (Botero, 2012).

\section{Tratamiento}

El régimen de elección consiste en pirimetamina (100-200mg de dosis inicial, después 50$100 \mathrm{mg} /$ día) + ácido folínico $10 \mathrm{mg}$ /día más sulfadiacina 2-8 g/día por vía oral mantenido durante al menos seis semana (Valdes M., 1996). Aunque esta combinación puede causar disminución de glóbulos blancos y problemas renales, es muy efectivo en contra de la toxoplasmosis. Más del 80\% de las personas muestran mejorías después de 2-3 semanas de tratamiento (Payen MC, 1997).

La más activa de las sulfamidas es la sulfadiacina, que se comporta de forma sinérgica con la pirimetamina, pudiendo además sustituirse por sulfonamidas triples (sulfameracina y sulfametacina) (Podzamczer D, 1998).

\section{REPORTE DE CASO}

Paciente masculino de 28 años de edad, con antecedentes de etilismo de 6 años de evolución y consumo de drogas ilícitas, fue ingresado el 12/10/2012 por presentar cefalea frontooccipital 
derecha de grado 7. Su historia revela vértigo y cefalea de una semana de evolución y una convulsión tonicoclónica con pérdida de la conciencia. Después de la crisis epiléptica el paciente refirió una sensación de adormecimiento del hemicuerpo izquierdo acompañado con vértigo, otalgia derecha y disartria. Una vez internado en el hospital la severidad de la hemiparesia fue aumentando y se agregó a la sintomatología diarrea con ausencia de pus, moco o sangre. En la exploración física se detectó hemiparesia de $2 / 5$ en el miembro superior izquierdo y de 3/5 en el miembro inferior izquierdo; también se observó la presencia de onicomicosis en todas las uñas de las manos y de los pies y papiledema. El estudio con Tomografía Axial Computarizada (TAC) reveló una lesión ocupante de espacio de $13 \mathrm{~mm}$ x $10 \mathrm{~mm}$ en el área subcortical del lóbulo parietal, con capsula formada y edema perilesional, sin comunicación con sistema ventricular; las cisternas perimesencefálicas estaban permeables (Fig. 1). Dentro de los antecedentes patológicos del paciente destacaban la amebiasis intestinal, neumonía, bronquitis y candidiasis orofaríngea, patologías secundarias al Virus de Inmunodeficiencia adquirida (VIH), diagnosticado en el año 2007. Se inició tratamiento con antirretrovirales (paciente había abandonado tratamiento hace tres meses), sulfadiacina, difenilhidantoina, fluconazol y ketoconazol. El paciente evolucionó satisfactoriamente al manejo médico y fue dado de alta después de su recuperación. 


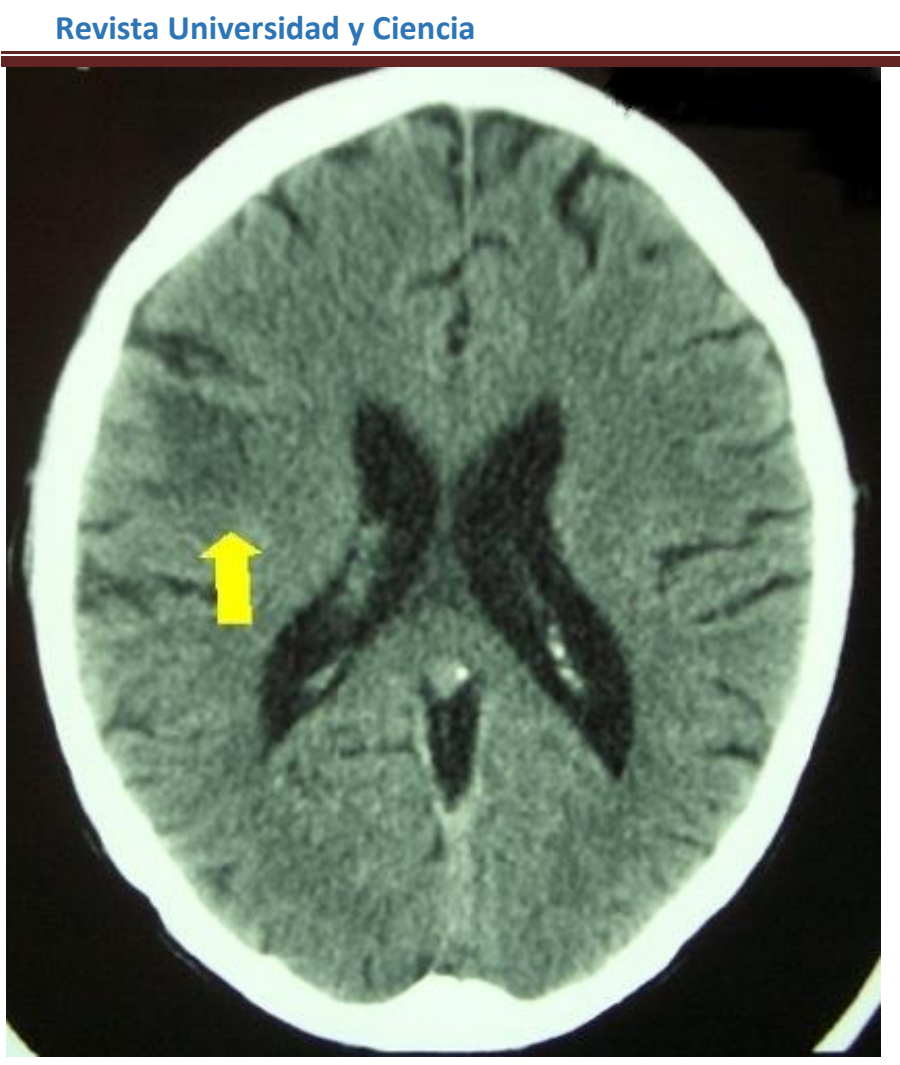

Fig. 1. Toxoplasmosis cerebral. TAC simple de hombre de 28 años de edad que presento cefalea, hemiparesia izquierda y convulsión; la imagen muestra lesión ocupante de espacio en el área subcortical del lóbulo parietal.

\section{DISCUSIÓN}

La toxoplasmosis asociada a $T$. gondii es una de las infecciones oportunistas más frecuentes del sistema nervioso central en pacientes con SIDA, llegando a ser la causa de 10 a 50\% de los casos (Perez Berrios J, 2004).Al ser éste un parásito intracelular obligado, se multiplica en células nucleadas, donde puede persistir durante años en forma de quiste, (Social, 2008) constituyendo un estado de infección latente, generalmente asintomática y que en pacientes con VIH puede reactivarse a razón del déficit inmune. Se ha publicado que más de dos tercios de estos pacientes tendrán un compromiso neurológico, y que la mitad de ellos podría desarrollar una masa intracraneal, (Social, 2008) reportándose hasta en un 10\% de los casos en los Estados Unidos de América y hasta un 50\% en Europa y África. La tendencia siempre ha sido que en estos pacientes se inicie una prueba terapéutica para toxoplasmosis en caso de presentar una lesión cerebral única. Para los pacientes sin mejoría clínica o radiológica bajo tratamiento por toxoplasmosis, se ha 
propuesto como método diagnóstico a seguir la biopsia cerebral abierta, ya que debemos recordar que el linfoma primario del SNC se encuentra también en estos pacientes, siendo de hecho la segunda causa más frecuente de lesiones cerebrales, sin olvidar también a la criptococosis.

En este caso, el diagnostico se confirmó con los estudios imagenológicos, con el cuadro clínico del paciente y con la epidemiologia, ya que él laboraba en una fábrica de ropa en donde convivía con decenas de gatos domésticos. El diagnostico de VIH se había hecho en el año 2007, lo que facilitaba la sospecha diagnóstica.

En nuestro medio, solo contamos con la tomografía axial computadorizada (TAC) como herramienta diagnóstica de imágenes en forma rutinaria; reservándose la resonancia magnética nuclear $(\mathrm{RMN})$ para casos que se consideren más complicados, de mayor dificultad diagnóstica desde el punto de vista de sospecha etiológica inicial y de su localización o extensión intracraneana.

Las lesiones toxoplásmicas parecen tener como características imaginológicas una localización más periférica, la presencia del signo del blanco excéntrico, ausencia de compromiso del cuerpo calloso, una pared uniforme, así como un marcado edema y la presencia de más de una lesión (Abosh A, 1991). De todas maneras, está documentada la similitud de los hallazgos imaginológicos en los pacientes con toxoplasmosis y linfoma, lo que dificulta su diferenciación (Hoffman J, 1993). La tomografía por emisión de positrones (SPECT por sus siglas en inglés) con el uso de talio-201, parece ser altamente específico para linfoma del SNC, pero poco sensible en casos de lesiones necróticas. Por otro lado, la tomografía por emisión de positrones (PET por sus siglas en inglés) si ha demostrado una gran utilidad para el diagnóstico y seguimiento del linfoma primario del SNC por ser ésta una lesión hipermetabólica (Rosenfeld S, 1992). Se realizó una búsqueda por medio del internet sobre otros casos en Nicaragua de presentación del SIDA con un absceso toxoplásmico único sintomático, cefalea y crisis motoras focales, no encontrándose otro reportado.

\section{Agradecimientos}

Al personal de la unidad de radiología e infectología del Hospital Escuela Dr. Roberto Calderón Gutiérrez-Managua, Nicaragua. Agradecemos especialmente a la profesora principal de 
microbiología y parasitología de la UNAN-Managua, Dra. Clara Isabel González Moncada, por monitorear el trabajo investigativo.

\section{REFERENCIAS}

Abosh A, P. L. (1991). Correlation Between Pre-operative Imaging Characteristics and Biopsy Diagnosis for Enhancing Intra-axial Lesions in AIDS Patients. AANS.org Libray Abstract, 15991.

Ammasari A, M. R. (1996). Toxoplasma gondii: An update on diagnosis and treatment. Springer , 209-222.

Ciricillo S., R. M. (1990). Use of CT and MR imaging to distinguish intracraneal lesions and to determine the need for biopsy in AIDS patients. J Neurosurg , 720-4.

Couvreur J., T. P. (1994). Manual de enfermedades infecciosas. In Infecciones obstetricas perinatales (pp. 160-81). Mexico: Mosby Doyma Libros.

David Botero, M. R. (2012). Parasitosis Humana. Medellin, Colombia: Corporacion para Investiagaaciones Biologicas.

Frenkel. (1997). Toxoplasmose. In Tratado de Infectología (pp. 1290-305). Sao Paulo: Atheneu.

Gongora-Biachi R. A., G.-M. (1998). Anticuerpos contra Toxoplasma gondii en pacientes con VIH en Yucatan. Rev Invest Clin , 419-22.

Hoffman J, W. H. (1993). Differentating Lymphoma from Nonmalignant Central Nervous System Lesions in Patients with AIDS. J Nucl Med, 567-575.

Ivonne Martin-Hernandez, S. M.-I. (2003). Toxoplasmosis: infeccion oportunista en pacientes con el sindrome de inmunodeficiencia adquirida. Rev. Biomed , 14:101-111.

Perez Berrios, L. C. (2004). Toxoplasmosis cerebral como manifestacion de Sindrome de Inmundeficiencia Adquirida (SIDA). Neuroeje , 52-55.

Llop, V.-D. Z. (2001). Microbiologia y Parasitologia Medicas Tomo III. Habana: Editorial Ciencia Medicas. 
Luft B. J. Hafner R., K. A. (1993). Toxoplasmic encefalitis in patients with adquired immunodeficiency syndrome. N England J Med , 995-1000.

McCabe R., C. V. (1993). Issues in toxoplasmosis. Infect Dis N A , 587-604.

Payen MC, D. S. (1997). A controlled trail of diapsone versus pyrimethamine-sulfadoxine for primary prophylaxis of Pneumocystis carinii, pneumonia and toxoplasmopsis in patients with AIDS. Biomed Pharm , 51:439-45.

Perez Berrios J, C. C. (2004). Toxoplasmosis cerebral como manifestacion de inmunodeficiencia adquirida (SIDA). Neuroeje , 52-55.

Podzamczer D, M. J. (1998). Thrice-weekly vs. daily sulfadiazine- pyrimethamine (SP) for maintenance therapy of toxoplasmosis encephlitis (TE). Conference on Retroviruses and Opportunistic Infections, Chicago, IL , 468.

Raffi F., A. J. (1997). A prospective study of criteria for the diagnosis of toxoplasmic encephalitis in 186 AIDS patientes. AIDS , 177-84.

Rina Liliana Luna Talavera, J. S. (2009). Toxoplasmosis cerebral asociada a VIH-SIDA:Reporte de un caso. Medigraphic, 283-285.

Rosenfeld S, H. J. (1992). Studies of Primary Central Nervous System Lymphoma with Fluorine18 fluorodeoxyglucose Positron Emission Topography J. Nucl Med , 532-536.

Social, M. d. (2008). Plan Nacional de Respuesta ante el VIH y el SIDA . ONUSIDA , 1-63.

Valdes M., D. A. (1996). Actualidades en el tratamiento y profilaxis de la toxoplasmosis. Rev Cubana Med Gen Inegr , 4-6. 